\title{
Fare storia contemporanea nell'età digitale: il caso italiano in chiave comparata
}

Tra il 16 e il 18 maggio 2013, presso il Dipartimento di economia e dell'impresa dell'Università degli studi della Tuscia (Viterbo), si è tenuto il convegno Una "nuova" storia contemporanea? Le riviste digitali e lo studio del passato. La redazione di "E-Review" ha chiesto a due relatori un resoconto dell'evento, domandando loro quale impatto stia avendo il diffondersi di esperienze digitali sull'uso pubblico della storia.

Between 16 and 18 May 2013, at the Department of economics and business at the University of Tuscia (Viterbo), it was held the conference A "new" contemporary history? Digital Magazines and the study of the past. The editorial staff of "E-Review" asked two speakers a report of the event, asking them what's the impact of the spread of digital experiences on the public use of history.

\section{Enrico Acciai: II mestiere dello storico verso una digitalizzazione transnazionale}

Il convegno viterbese - nato dalla collaborazione tra due riviste on line di studi storici, "Diacronie" e "Officina della Storia", con l'adesione della Sissco (Società italiana per lo studio della storia contemporanea, presente ai lavori con il proprio presidente Agostino Giovagnoli) e dell'Aiuci (Associazione per l'informatica umanistica e la cultura digitale) - è stato un appuntamento di cui si sentiva la necessità: nel corso dell'ultimo decennio il digitale si è imposto con forza nel mondo delle scienze umane e il successo delle riviste on line sta sancendo un cambiamento radicale nella diffusione delle ricerche e delle riflessioni storio- 
grafiche. Il mondo della ricerca storica è nel mezzo di un processo di radicale ridefinizione e a Viterbo questo è emerso con forza.

Basti pensare ai cambiamenti che ha conosciuto lo stesso mestiere dello storico, ormai proiettato verso una dimensione inevitabilmente transnazionale: un facile e rapido accesso on line tanto alle fonti primarie quanto alle riflessioni storiografiche più recenti sta contribuendo a una vera e propria rivoluzione della "professione". La comparsa di portali tematici, la digitalizzazione delle riviste cartacee, la messa in rete di archivi e biblioteche, la creazione di consorzi per l'accesso (a pagamento e non) alle riviste scientifiche, hanno infatti moltiplicato gli strumenti di lavoro a disposizione dello storico. Sia il linguaggio storico sia la forma in cui

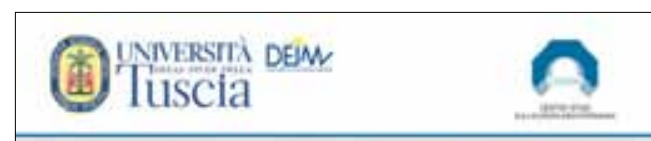

\section{Una "nuova" storia contemporanea?}

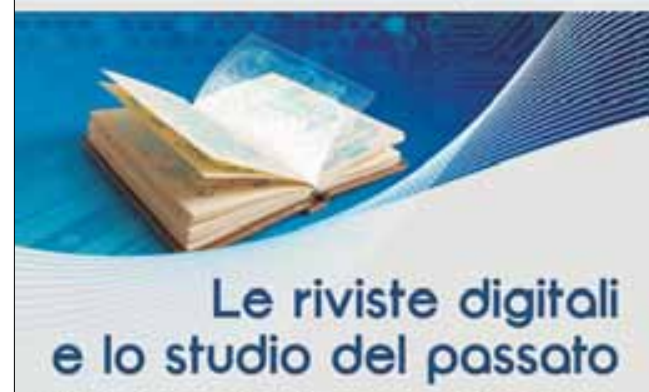

\section{Viterbo, 16-18 maggio 2013}

Università degli Studi della Tuscia Via 5. Maria in Gradi, 4

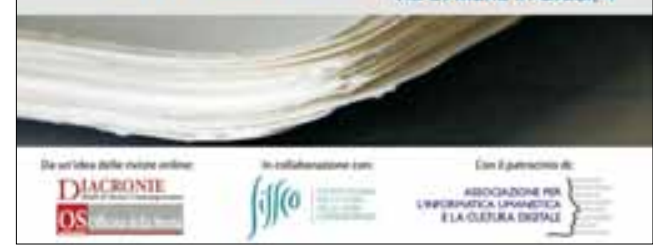

Locandina del convegno Una "nuova" storia contemporanea? Le riviste digitali e lo studio del passato comunicare storia si stanno quindi trasformando, seguendo così l'evoluzione delle società contemporanee. In tal senso la giovane redazione di "Diacronie" è quella che forse sta offrendo alcuni degli esempi più interessanti di utilizzo delle nuove tecnologie e dei social media. Dalla seconda parte degli anni Duemila il numero delle riviste storiche in digitale è progressivamente aumentato e, se si considera il panorama europeo nel suo complesso, quella digitale è una scommessa che sembra destinata a essere vinta. Sempre la redazione di "Diacronie" con la sua decisione di pubblicare contributi in più lingue ha offerto un chiarissimo segnale riguardo al carattere ormai transnazionale della produzione storiografica.

Nelle tre giornate viterbesi, gli interventi dei numerosi relatori coin-

volti hanno contribuito a dare un vero e proprio taglio seminariale al programma del convegno. Nel corso della prima sessione le relazioni di Tommaso Detti, Maurizio Ridolfi, Giancarlo Monina e Mirco Dondi hanno egregiamente introdotto il tema, ponendo l'accento sul percorso che ha portato all'attuale diffusione delle riviste digitali in Italia. La relazione di Monina è stata particolarmente utile 
perché capace di ricostruire alcune delle tappe più rilevanti del dibattito storiografico italiano sul digitale, dall'inizio degli anni Novanta. Dondi ha invece posto puntualmente l'accento sulla funzione sociale della ricerca storica e sulle enormi potenzialità legate alle nuove tecnologie.

La seconda giornata è stata dedicata alla presentazione dei diversi casi nazionali, con un focus privilegiato su quello italiano. Le relazioni hanno offerto notevoli spunti per riflettere sulla situazione delle riviste digitali nel nostro paese in prospettiva comparata. In tal senso sono stati particolarmente utili gli interventi di Elisa Grandi, che ha presentato il progetto editoriale della piattaforma revues.org, nato in Francia sotto il portale OpenEdition, e quello di Jacopo Bassi sul poco conosciuto caso dell'America latina.

L'ultima sessione è stata occupata da un workshop cui hanno partecipato Deborah Paci, Anna Caprarelli e i rappresentanti delle riviste "Storicamente" e "Storia e futuro", seguito da una tavola rotonda che ha tirato le fila dei lavori grazie ai puntuali interventi di Giovanni Fiorentino, Domenico Fiormonte, Serge Noiret e Stefano Vitali.

Nel corso delle tre giornate è emersa, tra molti relatori, l'esigenza di un superamento delle barriere esistenti tra le riviste storiche cartacee e quelle on line e di un rafforzamento del dialogo intergenerazionale riguardo al digitale. Il seminario ha inoltre cercato di far fronte all'esigenza di promuovere una riflessione sulle metodologie e sui problemi posti dalle contaminazioni tra strumenti e linguaggi diversi, destinati a integrarsi sempre di più nella teoria e nella pratica della ricerca, nella produzione storiografica e nel discorso pubblico sulla storia.

Volendo schematizzare ci sembra che i nodi irrisolti sul digitale emersi nel corso delle giornate viterbesi siano sostanzialmente tre. In primo luogo, si pone forte il tema della legittimità con cui la comunità scientifica "accoglie" i contenuti pubblicati sulle riviste digitali. La redazione di "Diacronie" ha ricordato come, in quattro anni di attività, non abbia ricevuto alcun contributo da ricercatori strutturati italiani, un dato che stride con la partecipazione di strutturati stranieri. In Italia persiste, evidentemente, un pregiudizio negativo nei confronti delle riviste non cartacee. Un problema che altrove, come hanno dimostrato Enrico Acciai e Matteo Tomasoni per il caso spagnolo, è stato in parte superato.

Il secondo nodo riguarda la volontà delle istituzioni pubbliche di scommettere chiaramente sul digitale. Com'è emerso dalla relazione di Gilda Nicolai e dalla riflessione conclusiva di Vitali, in Italia ci si trova ancora troppo spesso dinanzi un atteggiamento ambiguo da parte del "pubblico", in una generale mancanza di organicità nell'affrontare il tema del digitale. 
Il terzo e ultimo nodo è quello riguardante la proposta fatta, a nome di "Diacronie", da Paci. La redazione di "Diacronie" auspica, sostanzialmente, la nascita di un unico portale web di riviste digitali di scienze sociali italiane a libero accesso. Pur trattandosi di una necessità reale e stringente, anche considerando che in Europa l'Italia è ormai uno dei pochi paesi dove ancora non ci si è mossi in tale direzione, ci sembra che una proposta del genere meriti un'ulteriore riflessione e un confronto più approfondito tra i proponenti e le riviste on line di area umanistica in generale. Elemento dirimente, soprattutto in questo momento storico, sarà sicuramente quello economico. In virtù di questi tre nodi ci sembra che quello viterbese non possa che essere considerato come un primo incontro, sicuramente ben riuscito, di altri che si dovranno necessariamente organizzare nei prossimi mesi.

\section{Mirco Dondi: Dalla rete una sfida per la cultura accademica}

Uno degli aspetti salienti del convegno di Viterbo del maggio 2013 è stata la riflessione sulla storia e sul mestiere di storico dinanzi alla progressiva estensione del web. La rete non ci fornisce soltanto dati a disposizione, internet determina una cambiamento della natura della fonte mentre si ampliano i fruitori e si impone una profonda revisione degli strumenti necessari alla critica del documento. Da queste prime annotazioni, si deduce la indifferibilità del tema - e bene ha fatto la Sissco a prenderne atto - che presuppone mutamenti profondi nello statuto della disciplina.

I ricercatori, come gli insegnanti, sanno che la conoscenza non è mai data una volta per tutte, ma richiede un continuo aggiornamento. La rete enfatizza questo concetto dischiudendo l'accesso a documenti, informazioni, saggi che, ancora di più, accentuano quel senso di illimitatezza e inadeguatezza che alla vigilia del Novecento lo storico contemporaneista Charles Seignobos aveva lamentato. Lo storico contemporaneo - come in buona parte gli altri scienziati sociali - attua il principio di selezione delle fonti che si regge, nella fase di ricerca come nella stesura del testo, sulla capacità di elaborare criteri ordinatori che tuttavia non possono ormai prescindere dal rapporto fra il ricercatore e la rete. Non è un caso che il convegno viterbese, anziché proporsi con un titolo levigato e mediano sempre caro al linguaggio accademico, assesta la sua proposta di discussione in modo perentorio: Una "nuova" storia contemporanea?

Da questo punto di vista, le riviste digitali esprimono meglio le modificazioni in corso nella disciplina permettendo una circolazione che va oltre le biblioteche specializzate, garantendo l'uscita di contributi in tempi rapidi. Non ultimo, le riviste tendono sempre più a presentarsi come un luogo ideale di dibattito sui lavori 
terminati, su quelli in corso, e sui progetti di ricerca. Se ne deduce l'immagine di una potenziale vitalità con un'articolazione delle fonti che dallo scritto passa alla foto, all'immagine in movimento, al contributo audio per arrivare a una possibilità di riscontro della fonte offerta al lettore, mai conosciuta prima.

Naturalmente, le questioni poste sul tappeto hanno visto l'Italia in chiave comparata, dal momento che il web diventa un punto di confronto mondiale e le riflessioni sono in parte comuni. L'accesso alle nuove risorse digitali (limitandoci a portali scientifici, riviste e banche dati) non è disciplinato ovunque allo stesso modo e l'idea di open source che caratterizzerebbe un principio della rete è accolto solo in parte. Investimenti pubblici e libero accesso alle risorse caratterizzano $\mathrm{i}$ tratti delle iniziative avviate in Francia e in area ispanica. Il portale Latindex che accomuna America latina, Spagna e Portogallo raccoglie nel suo catalogo 21.000 riviste scientifiche digitalizzate. Dialnet, altro immenso riordinatore bibliografico di area ispanica, è nato nel 2006 e nel 2013 è registrato come il secondo portale bibliografico nel mondo. Rispetto a queste esperienze l'Italia manifesta apprezzabili iniziative (fra gli altri, Archivi del Novecento e la Rete degli archivi per non dimenticare), ma risente di una carenza di sviluppo - in termini quantitativi di materiali caricati - e riordinatrice a più alto livello istituzionale, anche a causa di minori investimenti rispetto agli altri modelli latini.

Vivace è il quadro delle riviste on line: per esigenze di tempo si sono privilegiate le esperienze di "Storicamente", "Storia e futuro" e "Diacronie", ma il quadro è reso ricco anche da altri contributi come "Officina della storia" - la cui redazione ha organizzato il convegno - o da ambiti specialisti come "Dep. Deportate, esuli e profughe". Non mancano esperienze emergenti come quella di "Percorsi storici" e siti di buona divulgazione scolastica o di riflessione sulla didattica della storia come "Clio92". Inoltre diverse riviste si stanno collocando a metà tra web e cartaceo, in un ottica - quella della rivista stampata - che complessivamente risulta sempre più difficile da gestire, alta nei costi e non più in grado di fornire quanto ora offre la rivista on line. Fotografato alla primavera 2013, il quadro è insomma assai mobile, anche per quanto riguarda le banche dati e le modalità di accesso. Altri due elementi sostanziano la rivoluzione digitale in corso: la tendenza ad accorpare la storia dentro al campo più generale degli studi umanistici, aprendosi a maggiori possibilità di interazione; e l'eventualità che internet possa modificare non soltanto la ricerca, ma anche l'organizzazione universitaria. Al riguardo, sono interessanti alcune tendenze che affiorano dalla Gran Bretagna, dove si chiede ai docenti di uscire dalle università per collaborare nei musei, ad esempio, oppure in programmi radiofonici o televisivi. La ragione di questo orientamento è legata a una nuova idea di visibilità che gli atenei cercano di sfruttare attraverso 
la notorietà dei propri professori. Nell'insieme però il modello britannico rimane contraddittorio: da un lato, per ciò che concerne la fruizione on line delle riviste, la realtà britannica rappresenta un modello in antitesi rispetto a quello italiano, $\mathrm{e}$ con maggiori criticità, poiché il libero accesso è limitato e gli editori propongono tariffe elevate che vanno a incidere sui costi delle ricerche, al punto da mettere in crisi le stesse biblioteche; dall'altro lato gli atenei cercano di dare risalto alla ricerca scientifica del corpo docente, al fine di acquisire prestigio e richiamare un maggior numero di studenti, ma è un modello di circolazione della conoscenza funzionale al marketing e non alla diffusione del sapere. Temi ampi quanto stimolanti, che investono il futuro della ricerca e di quello che sarà l'insegnamento universitario nei prossimi anni.

\section{Risorse}

Abstract del convegno:

http://storiaindigitale.wordpress.com/category/abstract

Videointerviste:

http://www.youtube.com/user/Storiaindigitale 